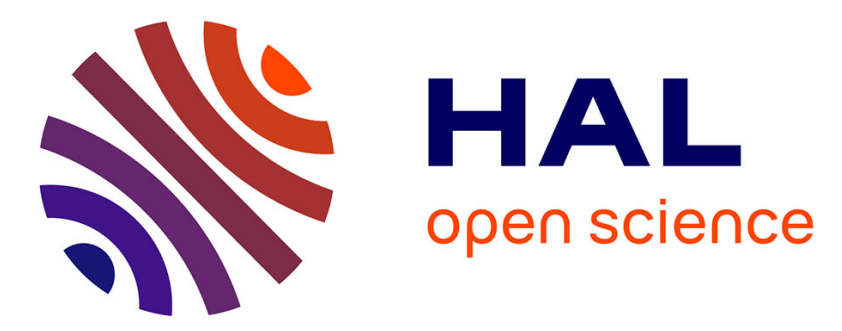

\title{
XI. Les schémas d'aménagement et de gestion des eaux en France. La SAGEsse s'est-elle imposée dans les politiques de l'eau? \\ Stéphane Ghiotti
}

\section{- To cite this version:}

Stéphane Ghiotti. XI. Les schémas d'aménagement et de gestion des eaux en France. La SAGEsse s'est-elle imposée dans les politiques de l'eau?. Droit et gestion des collectivités territoriales, DGCT (ancien annuaire des collectivités locales), 2010, 30 (1), pp.189-202. 10.3406/coloc.2010.2145 . hal02106275

\section{HAL Id: hal-02106275 \\ https://hal.science/hal-02106275}

Submitted on 23 Apr 2019

HAL is a multi-disciplinary open access archive for the deposit and dissemination of scientific research documents, whether they are published or not. The documents may come from teaching and research institutions in France or abroad, or from public or private research centers.
L'archive ouverte pluridisciplinaire HAL, est destinée au dépôt et à la diffusion de documents scientifiques de niveau recherche, publiés ou non, émanant des établissements d'enseignement et de recherche français ou étrangers, des laboratoires publics ou privés. 


\section{Les schémas d'aménagement et de gestion des eaux en} France. La SAGEsse s'est-elle imposée dans les politiques de l'eau ?

\section{Stéphane Ghiotti}

\section{Citer ce document / Cite this document :}

Ghiotti Stéphane. XI. Les schémas d'aménagement et de gestion des eaux en France. La SAGEsse s'est-elle imposée dans les politiques de l'eau ?. In: Droit et gestion des collectivités territoriales. Tome 30, 2010. Les enjeux de la gestion locale de l'eau. pp. 189-202;

doi : https://doi.org/10.3406/coloc.2010.2145

https://www.persee.fr/doc/coloc_2111-8779_2010_num_30_1_2145

Fichier pdf généré le 18/05/2018 


\section{LES SCHÉMAS D’AMÉNAGEMENT ET DE GESTION DES EAUX EN FRANCE}

\section{LA SAGEsSe s'est-elle imposée dANS LES POLITIQUES DE L'EAU?}

\section{Stéphane GHIOTTI}

I. LES SDAGE ET LES SAGE, RETOURS SUR LES FACTEURS DE LEURS ÉMERGENCES

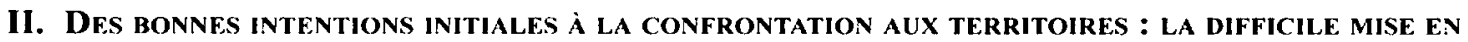
GUVRE DES SAGE

III. Quelles Perspectives ?

Bibliographie

La Loi sur l'eau de 1992 a créé deux nouveaux dispositifs de gestion de l'eau, les schémas directeurs d'aménagement et de gestion des eaux (SDAGE) et les schémas d'aménagement et de gestion des eaux (SAGE). Pour ce dernier, son caractère innovant, au regard des outils disponibles jusqu'alors au sein des politiques de l'eau, réside dans le fait qu'il s'appuie sur un triptyque combinant approche territorialisée, planification locale, concertation. L'avènement du SAGE soulève à l'époque un enthousiasme d'autant plus fort de la part des « acteurs de l'eau » qu'il s'insère parfaitement, au moins dans l'affichage, au sein d'un discours s'inspirant très largement des principes du développement durable tels que définis par les Conférences de Rio et de Dublin en 1992. Ces principes sont supposés par la participation, la décentralisation et la démocratie locale résoudre les conflits liés à l'environnement en général et à l'eau en particulier.

Avec la Directive-cadre européenne de 2000, transposée en droit français en 2004', qui oblige à l'atteinte du bon état écologique des eaux en 2015, la France est engagée dans un objectif de résultats. Les SAGE se retrouvent ainsi plus que jamais l'outil privilégié

1. Directive $2000 / 60 / \mathrm{CE}$ du 23 octobre 2000 établissant un cadre pour une politique communautaire dans le domaine de l'eau, transposée en droit français par la loi $n^{\circ} 2004-338$ du 21 avril 2004. 
des politiques de l'eau ce que confirme la Loi sur l'eau et les milieux aquatiques de 2006 (LEMA)', dernière loi sur l'eau en date complétant le dispositif national.

À l'aune de défis désormais fixés à l'échelle européenne, il n'est pas inintéressant d'analyser si le caractère innovant de ce dispositif a perduré, en s'interrogeant sur la manière avec laquelle il a répondu aux problématiques de l'eau des années 1990 et s'il est en mesure de répondre aux nouveaux enjeux et notamment d'articuler un cadre normatif européen, des problématiques nationales et des enjeux locaux.

\section{Les $S D A G E$ et les $S A G E$, retours sur les facteurs de leurs émergences}

Jusqu'à la Loi sur l'eau de $1992^{3}$, l'organisation d'ensemble de la politique de l'eau en France était régie par la première Loi sur l'eau en France de $1964^{4}$. Le dispositif institutionnel était conçu à l'échelle des six grands bassins hydrographiques des fleuves français ${ }^{5}$, chacun d'eux étant gérés par une instance de régulation politique, les comités de bassin appelés aussi «parlement de l'eau » et leur bras armé, les agences de l'eau. Ces dernières étaient chargées du financement d'une politique de l'eau avant tout centrée sur des problématiques de qualité (alimentation en eau potable et assainissement) en lien avec l'immédiat aprèsguerre. En effet, durant la période de reconstruction, les pressions sur les ressources furent considérables remettant en cause la pérennité de certains usages et débouchant par endroits sur des conflits voire des questions de santé publique. Ce système de financement, toujours en vigueur aujourd'hui, est basé sur la solidarité de bassin. Il s'appuie sur le prélèvement de redevances en fonction des volumes consommés et rejetés et leur redistribution sous forme d'aides reversées en application du principe pollueur-payeur. La loi prévoyait également la création d'une troisième instance, des établissements publics administratifs dotés de la maîtrise d'ouvrage opérationnelle, chargée de la mise en œuvre de la politique de l'eau. Si les comités et les agences de bassins se sont durablement installés dans le paysage institutionnel, les établissements publics n'ont jamais vu le jour rendant dès l'origine le système bancal. Un hiatus organisationnel et territorial s'est établi entre l'échelle des grands bassins et les problématiques locales.

Malgré les énormes efforts entrepris ${ }^{6}$ et les moyens investis pour lutter contre la pollution des eaux, l'intervention des pouvoirs publics est restée cependant très insuffisante de par l'éclatement des actions, le saupoudrage et le manque de moyens, l'absence de coordi-

2. Loi n ${ }^{\circ}$ 2006-1772 sur l'eau et les milieux aquatiques du 30 décembre 2006, JO du 31 décembre 2006.

3. Loi n' 92-3 du 3 janvier 1992 sur l'eau. JO du 4 janvier 1992.

4. Loi n"64-1245 du 16 décembre 1964. JO du 18 décembre 1964.

5. Les six agences de l'eau : Artois-Picardie, Adour-Garonne, Seine-Normandie, Rhin-Meuse, Rhône-MéditerranéeCorse, Loire-Bretagne.

6. Il est possible de noter le nombre de stations d'épuration construites notamment entre 1960, date à laquelle le tout à l'égout était généralisé, et 2004 où l'on dénombre 17302 unités. 
nation et un certain désintérêt politique à l'échelle locale (Valiron, 1990 ; Le Bourhis, 2004). Les cartes d'objectifs de qualité définies à l'échelle départementale restaient souvent le seul document de référence. La décennie 1980-1990 a marqué de ce point de vue un premier tournant dans ce domaine avec la mise en place d'outils contractuels locaux, les contrats de rivière. Ces derniers associaient pour une période de cinq ans l'État et les collectivités (mais pas les usagers privés de la rivière et les propriétaires riverains) autour d'un programme de financement de travaux visant pour l'essentiel l'assainissement et l'eau potable et dans une moindre mesure l'entretien des cours d'eau, ces derniers restant localement l'échelle de référence. Malgré des avancées certaines, le bilan de cette politique est resté en deçà des objectifs initialement fixés (Brun, 2003).

Une analyse critique de la situation est alors menée au sein du ministère de l'Environnement. Plusieurs constats ressortent et nécessitent à la fin des années 1980 de clarifier « les droits et les devoirs de chacun » et de reconfigurer les relations quadripartites entre l'État (la police de l'eau), les agences de bassin (le financement), les collectivités locales ayant pris de plus en plus de poids dans le cadre de la décentralisation, devenues de fait les opérateurs locaux enfin, les usagers " privés » de la rivière et les propriétaires riverains (Le Bourhis, 2004).

L'objectif des pouvoirs publics à cette période est d'adapter les cadres institutionnels organisationnels de la politique de l'eau en France ainsi que ses outils d'intervention afin de relever les nouveaux défis et enjeux (politiques, sociaux, environnementaux, financiers). Aux objectifs « historiques » de qualité s'ajoutent désormais ceux liés à la quantité et à la préservation des milieux aquatiques. La Loi sur l'eau de 1992 vise ainsi à mettre en œuvre une gestion globale et intégrée, assurant à la fois la satisfaction des besoins des usagers tout en assurant la protection des écosystèmes. Trois piliers soutiennent cette approche et caractérisent désormais la ressource en eau : sa patrimonialisation ${ }^{7}, 1$ '« unicité » de son régime juridique afin de renforcer la police de l'eau, la territorialisation de sa gestion.

Le statut patrimonial accordé à la ressource, le renforcement des pouvoirs de police de l'eau exercés par l'État, tout en affichant clairement la nécessité d'une gestion collective, répondent de manière nouvelle à des problématiques anciennes. Ces dernières sont inhérentes à ce que $\mathrm{P}$. Marc (2006) pointe comme étant une contradiction juridique de fond entre, d'un côté les cours d'eau relevant de la catégorie d'un bien affecté (les cours d'eau domaniaux et non domaniaux) et de l'autre les eaux courantes relevant d'une chose commune. L'usage et la gestion des cours d'eau en France oscillent ainsi statutairement entre deux vocations : l'une économique, l'autre sociale et patrimoniale, souvent difficilement conciliables. L'État ne pouvant prétendre à la domanialisation complète des eaux, malgré de nombreuses tentatives depuis près de deux siècles (Haghe, 1998), ni se résoudre à une gestion complètement privée, mobilise le principe d'une gestion collective d'un patrimoine commun pour contourner cette difficulté. Il favorise alors l'intervention d'un tiers « qui n'est ni public, ni

7. L'article 1 de la Loi sur l'eau de 1992 indique que l'eau fait désormais partie du patrimoine commun de la nation. Il s'agit d'un point novateur puisqu'il indique que la propriété privée n'est plus la seule garante de la gestion d'un bien. 
privé, mais quelque chose de l'ordre du communitas (plutôt que du sociétas), c'est-à-dire de la communauté d'usages de l'eau organisée localement autour d'une même ressource » (Barraqué, 1999, p. 381). Il s'agit en pratique d'abord de limiter, réduire les droits d'usages des riverains tout en augmentant le pouvoir de police sur les eaux en faveur des pouvoirs publics. Ce double mouvement vise à étendre leur contrôle, seul garant de l'intérêt général, en empiétant sur le domaine privé, les intérêts sectoriels, catégoriels, locaux (Césari, 2004).

La question de la territorialisation devient un des points centraux de la réforme. Les nombreux rapports parlementaires chargés de faire le bilan de la politique de l'eau durant les années 1980 notent une profonde dislocation des territoires de gestion des cours d'eau à l'échelle locale ainsi qu'une absence de planification. Il s'agit de trouver non seulement la bonne échelle de financement pour compléter (voire remplacer) l'action des agences mais aussi la bonne échelle d'intervention afin de favoriser localement la prise en charge des problématiques (la maîtrise d'ouvrage). S'il est souhaitable de réfléchir à un territoire de gestion commun, cette disposition ne serait suffisante sans un dispositif capable de coordonner les différentes opérations entre elles : la territorialisation et la planification sont indissociables. C'est dans cet esprit que la loi introduit par ses articles 3 et 5 deux nouveaux outils les SDAGE et les SAGE.

Le SDAGE fixe les grandes orientations et définit les grands principes d'utilisation de la ressource tant en quantité qu'en qualité à l'échelle des grands bassins hydrographiques :

«Les orientations fondamentales d'une gestion équilibrée de la ressource en eau [...]. Ils définissent de manière générale et harmonisée les objectifs de quantité et de qualité des eaux, ainsi que les aménagements à réaliser pour les atteindre. »

Il s'apparente au schéma de cohérence territorial (SCOT) en vigueur en urbanisme domaine avec lequel il interagit désormais « les programmes et les décisions administratives dans le domaine de l'eau doivent être compatibles ou rendus compatibles avec leur disposition. Les autres décisions administratives doivent prendre en compte les dispositions de ces schémas directeurs ». Cela témoigne de la volonté des politiques de l'eau de ne plus se contenter de gérer les flux d'eau (inondation) et de pollution une fois parvenus dans les cours d'eau. Il s'agit d'intervenir le plus en amont possible sur les espaces (urbain, agricole, touristique) $\mathrm{d}$ 'où proviennent ces flux en orientant autant que possible leur aménagement. Cela témoigne d'une innovation majeure au regard d'une organisation restée jusqu'alors marquée par la sectorisation poussée du modèle de gestion et la fragmentation des interventions. L'adoption du SDAGE marque en outre le choix de renforcer la place des agences de bassin au sein du dispositif au détriment des collectivités territoriales, le département notamment.

Les SAGE sont pensés comme un outil d'application et de planification locale du SDAGE au sein d'un périmètre pertinent d'un point de vue hydrographique (le bassin versant, nappe aquifère...) et socio-économique. Il fixe véritablement les règles d'utilisation et de partage collectif de la ressource entre tous les utilisateurs à l'intérieur de son périmètre. Le SAGE bénéficie d'une portée réglementaire puisqu'il est opposable aux autres décisions administratives dans le domaine de l'eau, ces dernières devant être conformes à ses 
dispositions. Il est ainsi beaucoup plus contraignant que le contrat de rivière et est dès lors considéré comme un « $\mathrm{PLU}^{8}$ hydraulique » :

«Dans un groupement de sous-bassins ou un sous-bassin correspondant à une unité hydrographique, à un système aquifère, le SAGE fixe les objectifs généraux d'utilisation, de mise en valeur et de protection quantitative et qualitative des ressources en eaux superficielles et souterraines et des écosystèmes aquatiques ainsi que de préservation des zones humides. »

Bien qu'il soit présenté comme un outil de gestion local, à l'initiative de ce dernier, le SAGE n'en demeure pas moins très encadré par la puissance publique puisque son périmètre doit être reconnu par un arrêté préfectoral. Sa structure politique, la commission locale de l'eau (CLE)", appelée aussi « parlement local de l'eau », est créée par le représentant de l'État en la personne du préfet coordinateur de bassin (Aspe, 1998). La question de la maîtrise d'ouvrage locale, chère au législateur depuis 1964, est également prévue par la loi par le biais de la communauté locale de l'eau. Pour faciliter la réalisation des objectifs arrêtés dans un SAGE, les collectivités territoriales intéressées et leurs groupements peuvent s'associer au sein de cette structure au statut d'établissement public. Elle établit et adopte un programme pluriannuel d'intervention après avis conforme de la commission locale de l'eau.

\section{Des bonnes intentions initiales à la confrontation aux territoires : la difficile mise en auvre des $S A G E$}

Les intentions du législateur sont claires. Il s'agit de remettre un peu d'ordre dans la politique de l'eau et de globaliser ses domaines d'intervention (qualité, quantité, environnement), d'encadrer politiquement et institutionnellement (notamment au niveau local) les conséquences de la décentralisation dans un domaine où l'État était depuis longtemps « le maître des ressources » et du jeu. Les outils proposés sont censés muscler les capacités d'intervention : police de l'eau, planification et portée réglementaire aux différentes échelles (bassin et locale) avec les SDAGE et les SAGE venant compléter les outils contractuels précédents. Ce « retour de l'État» comme le nomme Ch. Aspe (1998), n'a cependant pu se réaliser qu'à la faveur de la combinaison de plusieurs facteurs et en deux temps : il a fallu d'abord « imposer » la loi compte tenu de l'importance des changements envisagés, puis la mettre en œuvre.

Si la volonté politique de changement est réelle et le constat partagé quant à l'insuffisance de l'organisation précédente, notamment au sein de l'administration du ministère de l'Environnement, elle n'en demeure pas moins composite au sein de l'appareil d'État et particulièrement au sein des autres ministères (Agriculture, Équipement, Industrie,

8. Plan local d'urbanisme ayant remplacé les POS.

9. La commission locale de l'eau est formée à $50 \%$ par les collectivités territoriales, à $25 \%$ par les représentants de l'État et des établissements publics enfin $25 \%$ par l'ensemble des usagers. Elle est chargée de l'élaboration, de la révision et du suivi d'application du SAGE. 
Intérieur). Ces derniers vivent en effet très mal de voir s'accroître la sphère de compćtence et donc d'influence du ministère de l'Environnement (Lascoumes, 1999 ; Lascoumes, Le Bourhis, 1997). Le basculement des rapports de force intervient à la faveur des épisodes de sécheresse des années 1989-1990 (où l'agriculture irriguée est fortement remise en cause) et à partir de cette dernière date, la poussée des votes écologistes qui imposent avec force le champ de l'environnement sur la scène politique et médiatique (Vieillard-Coffre, 2001b). Des amendements au texte de loi initial afin de ménager les différentes susceptibilités finiront de « convaincre » les récalcitrants.

Le second niveau à convaincre fut les collectivités territoriales. Le caractère réglementaire et contraignant du SAGE laissait les élus inquiets de se voir imposer par l'État le financement et la mise en cuvre d'une politique de l'eau sur laquelle ils n'auraient que très peu de prise. Les débats portèrent notamment sur la composition de la CLE. Finalement, après plusieurs discussions lors des navettes parlementaires, le texte de loi fixe à $50 \%$ la part des élus locaux dans la CLE contre un tiers dans le projet initial du ministère de l'Environnement.

En complément de l'impulsion du ministère et de la pression sociale concernant la protection de l'environnement, le début des années quatre-vingt-dix bénéficie également d'un ensemble d'éléments, du local à l'international, favorable aux approches développées dans les SAGE (approche territoriale sur des bases naturelles, concertation, développement durable). En 1992, les processus de développement local se voient également dynamisés d'un point de vue politique après dix ans de décentralisation par l'adoption de la Loi relative à l'administration territoriale de la République (ATR) dite loi « Joxe ${ }^{10}$. Cette dernière renforce l'intercommunalité par la création de communautés de communes, d'agglomérations et urbaines et veut favoriser la démocratie locale. Depuis 1970, le statut du local est passé du « small is beautiful » au « small is efficient» (Malibeau, 1993, 1999). Plus qu'un changement de vocabulaire, cette évolution témoigne du changement de paradigme lié à l'aménagement et au développement, marqué par la valorisation sociale et économique des ressources environnementales locales. Ainsi, comme le rappelle G. Martin (1998), le retour du local s'explique autant par des raisons politiques, sociales qu'économiques.

1992 voit également l'adoption par la France du traité européen de Maastricht qui généralise le principe de subsidiarité défini comme le transfert du problème à gérer à l'échelle la plus adéquate pour le faire. C'est sur ce principe qu'émergent des territoires « cohérents » pour encadrer les dynamiques économique, démographique, environnementale, le plus souvent sur des bases fonctionnelles en décalage ou redécoupant les territoires administratifs de gestion traditionnelle des enjeux territoriaux. Les démarches des Pays (1995), des programmes LEADER ou encore les Parcs Naturels Régionaux (PNR) relèvent de ces principes bien que pour ces derniers leur existence soit antérieure.

Surtout, la généralisation des approches par bassin versant trouve sa reconnaissance politique, institutionnelle et scientifique à l'échelle internationale à la suite du Rapport Bruntland de 1987 (Ghiotti, 2001). La mise sur l'agenda politique de cette nouvelle approche

10. Loi n92-125 du 6 février 1992, JO du 8 fév. 1992. 
de gestion intégrée des ressources en eau (la GIRE) intervient et se renforce aux conférences de Dublin et de Rio de 1992 (Molle, 2010). À partir de cette date, les dispositifs nationaux s'inspireront et s'adapteront à cette approche, appuyés en cela par les bailleurs de fonds internationaux et les différents lobbys. Ces différentes institutions s'approprieront ce nouveau discours pour perpétuer, (re-) conquérir, affirmer leur légitimité et influer sur les considérables enjeux notamment financiers liés la problématique de l'eau (Ghiotti, 2005).

Près de quinze ans après leur création, il est difficile de véritablement porter un regard évaluatif complet sur un dispositif resté très longtemps quasiment lettre morte et qui, en 2005 ne comptait que 22 SAGE approuvés (pour 69 en cours d'élaboration et 13 en délimitation de périmètre) (Chiassérini, Pons-Serradeil, 2007). Une première tentative audacieuse - compte tenu du faible recul - fut menée par S. Allain $(2001,2002)$ après seulement une petite décennie de mise en œuvre, à partir d'un échantillon de dix cas et selon une approche comparative. Les observations contenues dans ce rapport pointent déjà les principales difficultés aujourd'hui rencontrées par ce dispositif. Elles se trouveront relayées et validées par d'autres études de cas plus locales (Landel, 2000) ou portant sur les structures animant les SAGE, les commissions locales de l'eau (CLE) (Le Bourhis, 1999). Elles montrent que la plupart des SAGE connaissent deux moments critiques. La mise en place difficile est liée à la phase de négociation et d'initialisation de la démarche. Des phénomènes d'interférence se font jour ensuite à partir du moment où se décide le démarrage du SAGE, c'est-à-dire l'élaboration de son contenu et l'affichage des objectifs.

Nous proposons ici de reprendre ces éléments d'analyse et de les mettre en perspective au regard « des temporalités » des SAGE sur une période de vingt ans entre 1990 et 2010 à l'aide de la base de données Gest'eau".

Sur la période 1988-2000, la politique des SAGE en France surfe véritablement sur cette vague porteuse du début des années 1990. Ce succès initial est cependant à relativiser par une concentration temporelle à laquelle correspond également une disparité spatiale, deux tendances qui rendent compte d'une difficile institutionnalisation, de mise en œuvre des SAGE en France. Près de $60 \%$ des signatures initiales (93 éléments sur un total de 156) interviennent entre 1988 et 2000 et plus particulièrement entre 1992 et 1998 où près de $50 \%$ $(48,7 \%)$ de ces engagements initiaux sont pris. La dynamique initiale des années 1992-1993 pour cet outil sera prolongée et accentuée par la définition des SDAGE définis entre 1996 et 1997 avec pour conséquence un pic de signatures (16) en 1998, niveau jamais atteint depuis (voir shéma). Autre indication, le délai entre l'engagement initial, correspondant à la période de sensibilisation et la phase opérationnelle (création des CLE) est relativement long puisqu'il faut en entre quatre et dix ans pour assurer cette transition. D'un point de vue spatial, les agences de l'eau Rhône-Méditerranée-Corse (RMC) et Loire-Bretagne (LB) apparaissent

11. Afin de dresser un état des lieux actuels, nous utiliserons des informations issues de la base de données SAGE du site internet Gest'eau (Gest'cau 2010). Cette dernière recense au 6 avril 2010,167 SAGE. Nous avons cn particulicr analysé deux éléments en son sein, la date de signature initiale du SAGE et la date de constitution de la CLE. Or, la base de données ne renseigne pas systématiquement ces différentes dates pour ces deux critères. C'est pourquoi, lorsque nous ferons spécifiquement référence à l'un d'entre eux, nous nous exprimerons à partir du nombre de SAGE renseignés, c'est-à-dire, un effectif total de 156 pour les signatures initiales et 137 pour la création de la CLE. 
comme les très bons élèves de cette politique des SAGE. Sur un total de 167 recensés au 6 avril 2010 - contre 187 en 2006 - les deux agences en totalisent $55 \%$ (23\% pour RMC et $32 \%$ pour LB), une place de leader confortée par le fait qu'elles abritent également le plus fort pourcentage de SAGE en première révision $(56 \%)$ et de mise en œuvre $(63 \%)$. Parmi les arguments explicatifs souvent mobilisés face aux difficultés, le caractère innovant de la procédure SAGE et son ambition sont souvent avancés. De par sa dimension locale, elle nécessite l'adhésion d'une diversité d'acteurs, l'implication des collectivités locales et le portage par un élu à la surface politique suffisamment large et solide pour fédérer mais aussi souvent imposer les décisions prises au sein de la CLE. Les questions de la maîtrise d'ouvrage locale et du financement de la politique de l'eau restent ainsi toujours au centre de la problématique. Elles nécessitent, pour leur dépassement, l'émergence d'une structure porteuse du SAGE suffisamment légitime puisqu'il s'agit de trouver un territoire politique en phase avec le territoire technique du bassin versant. Cette dernière dimension spatiale change d'ailleurs l'échelle spatiale dans l'appréhension des problématiques jusqu'alors envisagées depuis 1964 à l'échelle des cours d'eau. D'une approche centrée sur le zonage et la gestion sectorielle de la qualité de l'eau à une approche globale et territoriale, les outils de gestion locale de l'cau opèrent un renversement de paradigme. La finalité du projet n'est plus de gérer une rivière traversant un territoire mais de gérer un territoire traversé par une rivière où la ressource doit être partagée entre usagers.

Le SAGE propose ainsi un territoire (le bassin versant), une méthode (la concertation et la participation) et un mode de décision (la CLE) différents de ceux existant jusqu'alors. Il s'impose et s'insère dans un champ politique et de projet de territoire déjà existant qu'il bouleverse avec l'apparition d'un nouveau discours et d'une nouvelle norme concernant le partage des ressources. Son inscription territoriale devient problématique en faisant bouger voire remettre en cause les équilibres politiques, sociaux et territoriaux en place. Enfin, flanqué de sa dimension réglementaire, le SAGE est perçu comme un dispositif contraignant.

Cette difficulté est particulièrement flagrante à partir de 1998 où à la fois le nombre de signatures initiales et celui des constitutions de CLE chutent brutalement. À partir de cette date se fait jour une sorte de paradoxe. Alors que l'on observe un ralentissement dans « la naissance » de nouveaux SAGE - malgré une très légère reprise à partir de 2000 - à la même date, une implication plus forte dans la procédure apparaît de la part des porteurs de projets par une constitution significative de CLE. $60 \%$ d'entre elles sont signées entre 2001 et 2010. Ce relatif regain d'intérêt est cependant à tempérer car l'impulsion semble venir plus de la contrainte législative et réglementaire que de l'initiative locale. L'adoption par la France en 2000 de la DCE et sa transposition en droit français en 2004 a joué un rôle beaucoup plus déterminant que la LEMA adoptée en 2006 qui ne semble pas avoir relancé le processus. On retrouve ici le rôle majeur joué par les directives européennes sur les politiques nationales, notamment dans le domaine de l'environnement. 


\section{Schéma \\ La dynamique des $S A G E$ en France. Une longue et difficile institutionnalisation}

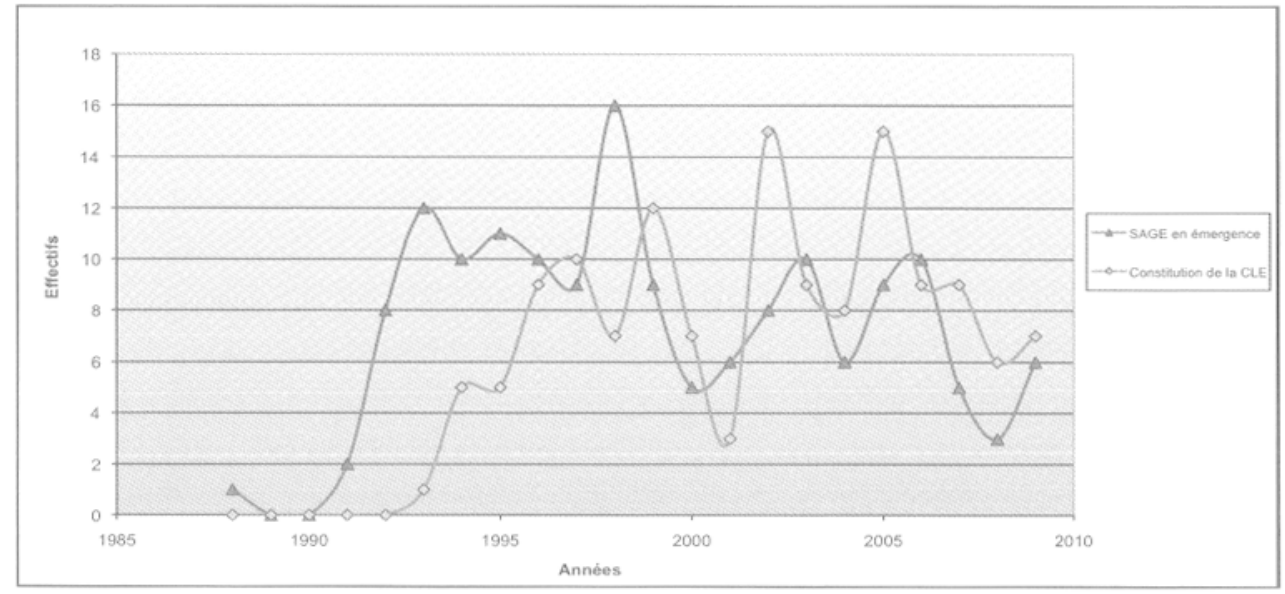

Source : Ghiotti, 2010 à partir du site Gest'eau

Le principal enjeu, mais aussi obstacle à la gestion locale d'un cours d'eau, est lié à sa dimension trans-territoriale alors qu'en parallèle, P. Billet (2005) observe au contraire, à la même échelle une profonde dislocation des différents territoires de gestion de ces cours d'eau. Cette tension entre processus intégratif et fragmentation apparaît comme une caractéristique majeure de l'action publique avec pour conséquence un déficit démocratique et d'efficacité (Ribot, 2007 ; Faure, Négrier, 2007). Le dispositif apparaît d'autant plus fragile que les réformes en cours (Révision générale des politiques publiques (RGPP), réforme des collectivités) n'apportent pas encore des éléments de réponse et d'orientation susceptibles de le renforcer et d'atteindre les objectifs fixés (Ghiotti, 2010).

\section{Quelles perspectives?}

Depuis 2000, plusieurs éléments d'ordre institutionnels et politiques ont témoigné de la reconnaissance du statut d'enjeux locaux dont relèvent les cours d'eau. Notons la Loi sur les risques de $2003^{12}$, qui consacre la reconnaissance d'un autre type de territoire de l'eau, les Établissement publics territoriaux de bassin, EPTB, (Marc, 2006). La loi de 2004 relative aux libertés et responsabilités locales ${ }^{1:}$ permet quant à elle la décentralisation du domaine public fluvial ou encore le transfert des anciennes concessions d'État (les Sociétés d'aménagement régional, SAR) aux collectivités territoriales. Enfin, l'adoption de la DCE en 2000 oblige à l'atteinte du bon état écologique des eaux en 2015. Il a ainsi été urgent de pouvoir

12. Loi $n^{\circ}$ 2003-699 du 30 juillet 2003 relative à la prévention des risques technologiques et naturels et à la réparation des dommages. $J O$ du 31 juillet 2003.

13. Loi n”2004-809 du 13 août 2004 relative aux libertés et responsabilités locales. JO du 17 août 2004. 
disposer d'un outil local de gestion de la ressource en état de marche pour l'appliquer et la mettre en œuvre. Cette nouvelle impulsion donnée aux SAGE était d'autant plus souhaitable que la LEMA l'a clairement positionné au centre du dispositif mais que ce dernier connaissait depuis 2005 un essoufflement notable tant du point de vue des engagements initiaux que de la constitution des CLE (voir graphique). Surtout, le SAGE, les CLE et les structures intercommunales qui les portent restent toujours aussi fragiles et souffrent d'une non-reconnaissance institutionnelle (en témoigne a contrario celle des EPTB) inquiétante à la vue des problématiques à gérer. Le double processus de décentralisation (transfert de compétence el charge) d'une part et d'augmentation du champ des contraintes (DCE, LEMA, RGPP...) d'autre part remet en cause la pérennité d'un édifice gestionnaire, reposant essentiellement sur des structures intercommunales, déjà fragilisées et non reconnues dans leurs missions, ces dernières débordant de plus en plus largement du champ d'intervention initialement défini.

Se fait ainsi jour une grande distorsion entre d'un côté les objectifs à atteindre, les coûts attenants et les réalités juridiques d'autre part (presque aussi variés que les problématiques à prendre en charge) qui fixent les compétences, les responsabilités, la visibilité institutionnelle, les outils et les moyens financiers et humains à disposition.

Si la décennie 1990 avait vu émerger avec force la place de l'environnement sur la scène sociale et politique, celle de 2000, débutée avec l'échec de la Loi Voynet sur l'eau, voit le contexte changer de manière radicale. Si l'intérêt pour l'environnement ne semble pas avoir fléchi au sein de la société, la place du ministère a en revanche considérablement évolué. La volonté et la nécessité maintes fois évoquées d'articuler eaux et aménagement du territoire au sein d'une approche globale s'est traduite d'un point de vue institutionnel par une réorganisation des contours et des missions du ministère en quatre temps et de manière extrêmement rapide. Entre 2007 et 2010 se sont ainsi succédés le ministère de l'Écologie et du Développement durable (MEDD en 2007), le ministère de l'Écologie, du Développement et de l'A ménagement durables (MEDAD en 2007), puis le ministère de l'Écologie, de l'Énergie, du Développement durable et de l'Aménagement du territoire (MEEDDAT, en 2008) et enfin le ministère de l'Écologie, de l'Énergie, du Développement durable et de la Mer (MEEDDEM en 2009). Si l'on peut effectivement louer l'idée de globaliser la gestion du territoire et de l'environnement (Énergie, climat, transport, logement, risques, mer, littoral), le ministère de l'Environnement est passé d'un statut de ministère d'État de plein droit à une sous-direction technique au sein d'un "super-ministère ». Si l'on peut s'inquiéter de voir restreint le champ de l'environnement à sa dimension technique, on peut également s'interroger sur la capacité de ses agents à se faire entendre en interne et à infléchir les rapports de forces en leur faveur lors des arbitrages. La longue histoire des relations entre ces différentes administrations (Haghe, 1998) et les grands corps qui les pilotent conjugués aux choix budgétaires concernant le recrutement de fonctionnaires ne laisse que peu de marge de manœuvre pour que la situation s'équilibre. À cela s'ajoute la profonde désorganisation/réorganisation des services, des personnels et de leurs missions avec une priorité donnée à l'échelle régionale au détriment du niveau départemental où l'on observe la disparition de l'ingénierie technique de l'État, la fusion des directions ministérielles déconcentrées. S'agit-il d'un développement ou d'un démantèlement durable de la question environnementale ? En tout état de cause, elle semble à nouveau soumise à un modèle de gestion où les contraintes multiples (financières, 
ressources humaines, réglementaires...) notamment au sein des SAGE, crispent les collectifs locaux qui voient les impositions/obligations s'accroître. Ces dernières devenant prioritaires et potentiellement conflictuelles ${ }^{14}$, cassent les dynamiques volontaires à l'œuvre au détriment de la gestion des milieux. Le paradoxe est alors total puisque, si la volonté de protection des milieux aquatiques n'a jamais été aussi forte, les dynamiques de gestion collective et concertée semblent devoir évoluer vers de l'administration des obligations réglementaires. L'initiative locale et la proximité avec les problématiques de terrain se retrouvent pour partie court-circuitées avec ces nouvelles orientations. Au sein de ce système, la connaissance et l'expertise deviennent des atouts/enjeux majeurs de la décision mais se retrouvent de plus en plus externalisées à la puissance publique en direction de mouvements associatifs, catégoriels et de bureaux d'études privés.

Une nouvelle période de tension apparaît donc entre quatre groupes d'acteurs. L'État responsable de ses engagements devant l'Europe et ses opérateurs, telles les agences confrontées au double problème des contraintes budgétaires et de difficulté à trouver des partenaires locaux. Le troisième groupe est constitué des collectivités territoriales dont le pouvoir et les responsabilités se sont accrus et l'investissement financier devenu majeur dans le domaine de l'environnement (Ifen, 2002, 2003, 2005). Cependant, elles sont touchées par la réforme engagée par le gouvernement de François Fillon visant la recomposition de leurs compétences et de leurs modalités d'élection. Cette réforme les inquiète car elle touche à l'existence même du département et/ou leurs capacités politiques et financières. Enfin, les usagers de l'eau qui voient s'opérer un renversement dans la hiérarchie des usages avec la priorité donnée au milieu.

La redéfinition des relations entre ces groupes (bien sûr, loin de constituer des blocs homogènes) participe à celle entre socićté ct cnvironncment et fixe les enjeux autour de quatre dimensions : la dimension scientifique qui place la connaissance, l'expertise et la capacité d'innovation au centre des processus de gestion et de décision ; la dimension politique avec une nouvelle étape dans les débats visant l'organisation et le contrôle du territoire ; la dimension sociale par la recomposition des jeux d'acteurs à travers la nouvelle hiérarchisation des priorités au sein du système des usagers de la ressource; enfin la dimension spatiale avec la nécessité d'identifier une nouvelle échelle de gestion.

À l'issue des nombreuses évolutions législatives de la décennie 2000 et des nouveaux enjeux, le constat d'un double hiatus organisationnel et territorial apparaît, rendant toujours difficile l'émergence d'une maîtrise d'ouvrage locale susceptible notamment de porter les SAGE. Le hiatus territorial se fait jour entre une gouvernance à l'échelle des grands bassins, opérée par le comité de bassin et les structures de gestion locale des cours d'eau.

14. Prenons l'exemple de l'article 6 de la LEMA qui impose à l'horizon 2014 une multiplication par quatre des débits réservés pour les ouvrages prélevant en rivière (du 1/10 au 1/40 du module). Cette situation est particulièrement conflictuelle en milieu méditerranéen avec la nécessaire redéfinition du partage de l'eau entre agriculture, tourisme, alimentation en eau potable et milieux naturels. Un débat majeur s'engage ces derniers mois pour le mode de gouvernance et la réaffectation des « économies d'eau » réalisées. Rappelons pour mémoire, que les cours d'eau méditerranéens ont pour leur quasi-totalité des débits moyens annuels inférieurs au 1/10 du module. Cette situation pose la question de la pertinence des concepts utilisés pour atteindre les objectifs de bon état. 
Il est complété par un hiatus organisationnel pour ces structures de bassin dont l'enjeu est d'être pérennisées (juridiquement et financièrement) au regard des objectifs à atteindre et des moyens propres disponibles et alloués pour y faire face. Ces structures se retrouvent « coincées » entre les EPTB (situés à une échelle interdépartementale ou interrégionale) et les structures intercommunales (types communautés de communes). Une proposition émerge au sein de la région Provence-Alpes-Côte d'Azur par le biais d'un groupe de travail animé par l'Agence régionale pour la protection de l'environnement (ARPE) dont les premiers résultats des réflexions émergent en 2009. La solution proposée pour renforcer et "muscler » les structures de gestion locale de l'eau s'articule autour de la notion clé d'Établisscment Public d'Aménagement et de Gestion des Eaux (EPAGE). Elle se singularise par son caractère innovant mais aussi par rapport à son positionnement à contre-courant des dynamiques de réformes qui tendent plutôt à une simplification de la carte territoriale.

À côté de cet écueil majeur, la reconnaissance de l'EPAGE est difficile car il se pose la question de savoir comment et sur quelle base se fixent les limites entre ce qui doit définir le périmètre d'intervention et répondre ainsi aux objectifs et ce qui en est exclu. Un équilibre est à trouver entre le fait de ne pas rentrer en concurrence avec l'existant (les EPTB notamment), avoir les moyens d'agir et ne pas trop s'exposer juridiquement au risque que la « rationalisation du territoire de l'eau »n'ouvre la porte à des contentieux conséquents.

S'il est trop tôt pour tirer des conclusions hâtives des éventuelles conséquences des lois Grenelle 2 et celles liées à la réforme des collectivités, l'ampleur des enjeux actuels et à venir et leur non-prise en compte (a minima que se passe-t-il en cas d'incapacité des structures de gestion existantes d'assurer leurs missions ?) semblent militer pour une réponse des pouvoirs publics.

Dans le contexte actuel, les SAGE apparaissent nécessaires mais non suffisants à la conduite actuelle et prochaine de la politique locale de l'eau. Même si son caractère réglementaire est renforcé par la LEMA par le biais de la reconnaissance de son opposabilité au tiers, un relatif assouplissement du dispositif est cependant à noter (disparition de la notion de prise en compte). Il est possible d'interpréter cette évolution comme un compromis entre le nécessaire renforcement des pouvoirs de police de l'État au regard de sa responsabilité face à l'Union européenne et les pouvoirs locaux, plus puissants, plus impliqués mais ayant connu des difficultés à initier la démarche. Plus que le dispositif lui-même et son fonctionnement, il apparaît que les moyens de son insertion territoriale par sa reconnaissance institutionnelle et son renforcement juridique et économique soient les conditions préalables indispensables à l'atteinte des objectifs.

\section{Bibliographie}

Allain S., " Planification participative de bassin et gouvernement de l'eau », in Les territoires de la participation, Géocarrefour, vol. 76, n 3, 2001, p. 199-209.

Al.taIn S., La planification participative de bassin. Analyse des conditions d'émergence et des modalités d'élaboration des Schémas d'Aménagement et de Gestion des Eaux 
$(S A G E)$, Rapport final de synthèse $n^{\circ} 1$, Groupe inter-bassin SDAGE-SAGE, INRAENGREF, 2002, 174 p.

ASPE Ch., « Le retour de l'État et l'argumentaire scientifique dans les conflits pour l'eau », in Régulation de l'eau en milieu méditerranéen, risques et tensions, Territoires en Mutation, $\mathrm{n}^{\circ}$ 3, 1998 (mars), p. 83-94.

Barraqué B., «Entre public et privé, l'eau et ses agences », in M. Marié, D. Larcena, P. DÉRIOz (dir.), Cultures, usages et stratégies de l'eau en Méditerranée occidentale, tensions, conflits et régulations, Paris, L'Harmattan, coll. «Villes et Entreprises », 1999, p. 371-396.

Billet P., « La gestion locale des cours d'eau dans le contexte d'unification de la directive 2000/60 du 23 octobre sur l'eau ", in La gestion locale des cours d'eau, Droit de l'environnement, $\mathrm{n}^{\circ}$ 125, numéro spécial, 2005, p. 18-22.

Brun A., Les politiques territoriales de l'eau en France. Le cas des contrats de rivières dans le bassin versant de la Saône, thèse de géographie, INA P-G, 2003, 376 p.

CÉSARI S., "La nouvelle " police de l'eau et des milieux aquatiques. Ingénieurs et techniciens de l'État dans la mise en xuvre de l'action réglementaire (1992-1998), doctorat de Science Politique, IEP Grenoble, Université Pierre Mendès France, 2004, $484 \mathrm{p}$.

Chiassérini V., Pons-Serradeil. M., « Les nouveaux schémas d'aménagement et de gestion des eaux : de l'orientation à une subtile contrainte ? », Bulletin du Droit de l'Environnement Industriel, supplément au no 12, 2007, p. 11-17.

Faure: A., Négrier E. (dir.), Les politiques publiques à l'épreuve de l'action locale. Critiques de la territorialisation, Paris, l'Harmattan, 2007, 302 p.

Guortı S., Les territoires de l'eau. Gestion et développement en France, Paris, cNRS EDITIONS, coll. «Espaces et Milieux », 2007, 246 p.

IFEn, Les dépenses d'environnement des Départements et des Régions (1996-2002), Les dossiers, $\mathrm{n}^{\circ} 2,2005,24 \mathrm{p}$.

IFEN., Les dépenses des Régions en matière d'environnement, les données de l'environnement, $\mathrm{n}^{\circ} 82,2003,4 \mathrm{p}$.

IFen., Les dépenses des Départements en matière d'environnement, les données de l'environnement, $\mathrm{n}^{\circ}$ 79, 2002, $4 \mathrm{p}$.

LANDEl P-A., « Gestion de l'eau et recomposition territoriale. L'exemple de la rivière Drôme », in Eau, territoire et développement, REM, vol. 49, n'194-195, 2000, p. 133-144.

Lascoumes P., l'Eco-pouvoir, environnements et politiques, La Découverte, Paris, 1994, $320 \mathrm{p}$.

Lascoumes P., Le Bourhis J-P., L'environnement ou l'administration des possibles, la création des directions régionales de l'environnement, Paris, L'Harmattan, 1997, 253 p. LAscoumes P. (edit), Instituer l'environnement, vingt-cinq ans d'administration de l'environnement, L'Harmattan, 1999, 233 p. 
Le Bourhis P., « La publicisation des eaux. Rationalité et politique dans la gestion de l'eau en France (1964-2003) ». Thèse pour le doctorat es science politique, Paris-I Panthéon-Sorbonne, 2004, 529 p.

LE Bourhis P., « Les Commissions Locales de l'Eau, quelques remarques sur la mise en cuvre d'un dispositif de planification négocié », in M. MARIE, D. LARCENA, P. Derioz (dir.), Cultures, Usages et Stratégies de l'eau en Méditerranée Occidentale, tensions, conflits et régulations, Paris, L'Harmattan, coll. «Villes et Entreprises », 1999, p. 519-533.

Malibenu A. (dir.), À la recherche du local, Paris, L'Harmattan, coll. « Logiques politiques », 1993.

Malibeau A., « Le local comme phénomène de société (2). Le local et ses représentations », in Entreprises et Territoires, Sciences de la société, n 48, 1999, p. 197-220.

MARTIN G., "Le territoire introuvable », in MARTIN G. (dir.), La dynamique des politiques sociales. Observation, management, évaluation, Paris, L'Harmattan, 1998, p. 196.

MARC P., Les cours d'eau et le droit, Paris, Éditions Johanet, 2006, 292 p.

Molle F., "La GIRE : Anatomie d'un concept », in Julien F. (ed), Gestion intégrée des ressources en eau : paradigme occidental, pratiques africaines, Chap. 2, Québec, Presses de l'Université du Québec, 2010, (à paraître).

Riвот C., " Les collectivités territoriales et l'eau : dispersion des actions et éloignement des acteurs ", Bulletin du Droit de l'Environnement Industriel, supplément au $\mathrm{n}^{\circ} 12$, 2007, p. 31-34.

Valiron F. (dir.), La Politique de l'eau en France de 1945 à nos jours, Paris, Presse de L’École Nationale Des Ponts et Chaussées, 1990, 149 p.

Vieill.ard-Coffre S., « Gestion de l'eau et bassin versant », in Géopolitique de l'eau, Hérodote, Paris, La Découverte, n 102,2001a, p. 139-156.

VIEILlARD-COFFre S., « Les Verts et le pouvoir. Regards géopolitiques sur les écologistes français : stratégies et représentations », in Écologie et géopolitique en France, Hérodote, Paris, La Découverte, n*100, 2001b, p. 120-150.

http://www.gesteau.eaufrance.fr/, consulté le 6 avril 2010. 